\title{
Survey of bryophytes in Serra da Canastra National Park, Minas Gerais, Brazil
}

\author{
Dimas Marchi do Carmo ${ }^{1 *}$ and Denilson Fernandes Peralta'
}

Received: September 9, 2015

Accepted: March 23, 2016

\begin{abstract}
Serra da Canastra National Park is located in southwestern Minas Gerais State and contains several phytophysiognomies of the Brazilian Cerrado. To date, no study on bryophytes has been conducted in this area and the present study is the first to be carried out in this major biological reserve of Cerrado located on the Brazilian Plateau. This study found 289 species of bryophytes, including mosses, liverworts, and one species of hornwort, representing fourtyone Brazilian endemic species and 56 new records for Minas Gerais State. Most species are widely distributed in Brazil, with only $16 \%$ having restricted distributions. Regarding worldwide distribution, $31 \%$ are Neotropical. Lejeuneaceae had the highest species richness among liverwort families with 53 species, whereas Sphagnaceae had the highest richness among moss families with 26 species. Phaeoceros laevis was the only hornwort species found in the park. This study contributes to the understanding of bryophyte species richness and distribution, and provides the worldwide and Brazilian distribution of the bryophyte flora of Serra da Canastra National Park, an important center for biodiversity conservation.
\end{abstract}

Keywords: cerrado, floristic, hornwort, liverworts, mosses, mountain areas

\section{Introduction}

Serra da Canastra National Park was created in order to protect the source of the São Francisco River, which is the watercourse arising in the huge chest-shaped plateau. Serra da Canastra divides two watersheds: the south portion is the beginning of the Paraná River and the north portion is the beginning of the São Francisco River (Souza \& Costa 2011).

The predominant vegetation is represented by grasslands and rocky fields (campos rupestres) (Carvalho-Silva \& Guimarães 2009), which ensure a high degree of endemism since they occur in areas with particular ecological conditions influenced by geographic isolation and biotic and abiotic factors (Harley \& Simmons 1986; Giulietti et al. 1987; Pirani et al. 1994; Stannard 1995). Since the park is open to visitors there is some anthropic disturbance, such as the installation of unpaved roads and buildings; nevertheless, there is a relatively high degree of endemism among some angiosperm species, and so a great need for the permanent preservation of the area as a conservation unit (Romero \& Nakajima 1999).

Floristic surveys conducted in areas of Minas Gerais that are similar to Serra da Canastra include Yano \& Carvalho (1995) who found 46 species in Serra da Piedade; Yano \& Peralta (2009) who found 42 species of mosses and hepatics in the Grão-Mogol mountains; Yano \& Peralta (2011a) found 114 species in Serra de São José at the city of Tiradentes; Yano \& Peralta (2011b) found 237 species in Serra do Cipó; Luizi-Ponzo et al. (2013) found 209 species in Ibitipoca State Park and, recently, Souza \& Câmara (2015) who studied the mosses of gallery forest in Serra do Cipó National Park. These works contain relevant floristic data and are useful in the

\footnotetext{
${ }^{1}$ Instituto de Botânica, Núcleo de Pesquisa em Briologia, P.O. Box 68041, 04045-972, São Paulo, SP, Brazil

* Corresponding author: dimas.botanica@gmail.com
} 
identification of some species. Furthermore, they provide species lists and document the location of bryophytes in these regions, resulting in knowledge of about 766 species of bryophytes in Minas Gerais State (Costa \& Peralta 2015).

These works notwithstanding, floristic inventories of bryophytes in mountainous areas of Minas Gerais are still sparse considering the extensive area of the Cerrado domain in the state and in comparison to other terrestrial plant groups. Therefore, Serra da Canastra is an interesting area with great potential for the study of bryophytes (Costa et al. 2011). Furthermore, there are few compilations of the occurrence of Brazilian bryophytes among phytogeographic domains that also include their distribution within Brazil and worldwide.

To date there has been no study of the bryophytes in this significant biological reserve of Minas Gerais. Therefore, this study aimed to carry out a floristic survey of the bryophytes in Serra da Canastra National Park by analyzing bryophyte species richness, occurrence among phytogeographic domains and Brazilian and worldwide geographical distributions.

\section{Materials and methods}

\section{Study area}

The Serra da Canastra National Park is situated on a mountain range located in southwestern Minas Gerais and encompasses parts of the municipalities of Sacramento, São Roque de Minas and Delfinópolis (20 00'-20³0'S and $46^{\circ} 15^{\prime}-47^{\circ} 00^{\prime} \mathrm{W}$ ). It contains 71,525 ha with average altitude ranging from approximately 800 to $1,200 \mathrm{~m}$, with the highest point of the park being Serra Brava at 1,496 m. The average temperature is around $17^{\circ} \mathrm{C}$ in the winter and $23^{\circ} \mathrm{C}$ in the summer, but varies between higher and lower regions. Average annual rainfall ranges from approximately 1,300 to $1,700 \mathrm{~mm}$, with most rainfall being concentrated from December to February (IBDF 1981).

There are several phytophysiognomies in Serra da Canastra National Park, which provide many conditions for the development of bryophytes, such as rocky fields (campos rupestres), rocky cerrado (cerrado rupestre), grasslands , "dirty fields" (campos sujos), cerrado sensu stricto, gallery forests (matas de galerias), slope forests (matas de encosta), moist fields (campos úmidos), and waterfall areas (Couto Júnior et al. 2010).

\section{Data collection and identification}

Twenty samples from the study area were found in SP herbaria (Holmgren et al. 1981) and four one-week long expeditions were made to the park during to make collections in 2012 to 2014.

Collections were made by walking freely through the main phytophysiognomies of the park including waterfalls, rocky fields (campos rupestres), gallery forest (matas de galeria) and slope forest (matas de encosta) (Couto Júnior et al. 2010). Substrates suitable for bryophyte colonization were classified according to Robbins (1952) and Bates (2009) with some modifications. The methodology for collection, herborization and preservation of material followed Gradstein et al. (2001), and all samples collected were deposited in SP.

Identification of the species followed Frahm (1991), Sharp et al. (1994), Yano \& Carvalho (1995), Buck (1998), Vilas Bôas-Bastos \& Bastos (1998), Bastos et al. (2000), Gradstein et al. (2001), Castro et al. (2002), Gradstein \& Costa (2003), Câmara \& Costa (2006), Câmara (2008a; b), Ballejos \& Bastos (2009), Yano \& Peralta (2009), Valente et al. (2011), Yano \& Peralta (2011a; b), Bordin \& Yano (2013), Luizi-Ponzo et al. (2013) and Valente et al. (2013).

The classifications followed herein are those of Renzaglia et al. (2009) for Anthocerotophyta, Crandall-Stotler et al. (2009) for Marchantiophyta and Goffinet et al. (2009) for Bryophyta.

The list of species is organized in alphabetical order by family, then genus and then species, and provides the Brazilian geographical distribution as proposed by Valente \& Pôrto (2006), who considered as "rare" those bryophythes occurring in one to four Brazilian states; as "moderate" those occurring in five to nine states; and as "wide" those occurring in ten or more Brazilian states. The distribution and biogegraphical domains followed Gradstein \& Costa (2003), Yano (2008), Forzza et al. (2010), Costa et al. (2011) and Costa \& Peralta (2015).

\section{Results and discussion}

\section{Species richness}

One thousand one hundred and seventy-seven $(1,177)$ samples were analyzed, with 722 samples of mosses (Bryophyta), 454 of liverworts (Marchantiophyta) and only one of a hornwort (Anthocerotophyta). Two hundred and eight-nine species of bryophytes were found, representing 128 genera and 57 families, accounting for approximately $38 \%$ of the species estimated for the state and $19 \%$ for the country (Tab. 1) (Costa \& Peralta 2015).

The family Lejeuneaceae was the most represented with 53 species (18\%). The number of moss species was greater (174 species) than that of liverworts (114 species), while only one species of hornwort was found (Tab. 1). Among mosses (Bryophyta) the most common families were Sphagnaceae, with 26 species, followed by Leucobryaceae (21 species), Bryaceae (13 species) and Sematophyllaceae (12 species). Among liverworts (Marchantiophyta), the most common family was Lejeuneaceae, with 53 species, followed by Lepidoziaceae (10 species) and Frullaniaceae 
Table 1. List of species found in Serra da Canastra National Park. Phytogeographic domains (Phyt. dom.): Amazon Rainforest = AM, Atlantic Forest $=$ AT, Cerrado $=\mathrm{CE}$, Caatinga $=\mathrm{CA}$, Pampa $=$ PA, Pantanal $=$ PL; Geographical distribution of Brazil $($ Brazil distr. $)$; Geographical distribution worldwide (World distr.); * New record for Minas Gerais State.

\begin{tabular}{|c|c|c|c|c|}
\hline Species & Phyt. Dom. & Brazil distr. & World distr. & Voucher \\
\hline \multicolumn{5}{|l|}{ Anthocerotophyta } \\
\hline \multicolumn{5}{|l|}{ NOTOTHYLADACEAE } \\
\hline Phaeoceros laevis (L.) Prosk & $\mathrm{AM}, \mathrm{AT}, \mathrm{CE}, \mathrm{PA}, \mathrm{PL}$ & Wide & Subcosmopolitan & Peralta 15167 \\
\hline \multicolumn{5}{|l|}{ Marchantiophyta } \\
\hline \multicolumn{5}{|l|}{ ACROBOLBACEAE } \\
\hline $\begin{array}{l}{ }^{*} \text { Tylimanthus laxus (Lehm. \& Lindenb.) Steph. } \\
\text { ANEURACFAF }\end{array}$ & AT, CE, PL & Moderate & Tropical America & Peralta 15198 \\
\hline ANEUKACEAE & AM, AT, PL & Wide & Subcosmopolitan & Peralta 15027 \\
\hline Riccardia cataractarum (Spruce) Schiffn. & $\mathrm{AT}, \mathrm{CE}, \mathrm{PL}$ & Wide & Tropical America & Carmo 1008 \\
\hline Riccardia digitiloba (Spruce ex. Steph.) Pagán & $\mathrm{AM}, \mathrm{AT}, \mathrm{CE}, \mathrm{PL}$ & Wide & Tropical America & Peralta 15703 \\
\hline Riccardia regnellii (Angstr.) Hell & $\mathrm{AT}, \mathrm{CE}$ & Wide & Endemic to Brazil & Carmo 415 \\
\hline \multicolumn{5}{|l|}{ BALANTIOPSIDACEAE } \\
\hline Isotachis multiceps (Lindenb. \&Gottsche) Gottsche & $\mathrm{AT}$ & Moderate & Tropical America & Carmo 839 \\
\hline Isotachis serrulata (Sw.) Gottsche & $\mathrm{AM}, \mathrm{AT}$ & Moderate & Tropical America & Carmo 896 \\
\hline Neesioscyphus argillaceus (Nees) Grolle & $\mathrm{AT}, \mathrm{CE}$ & Moderate & Tropical Andes and Brazil & Carmo 1037 \\
\hline Neesioscyphus homophyllus (Nees) Grolle & AT & Rare & Argentina and Brazil & Carmo 948 \\
\hline \multicolumn{5}{|l|}{ CALYPOGEIACEAE } \\
\hline Calypogeia grandistipula (Steph.) Steph. & AT & Rare & Endemic to Brazil & Carmo 802 \\
\hline Calypogeia laxa Gottsche \& Lindenb. & $\mathrm{AM}, \mathrm{AT}$ & Wide & Tropical America & Peralta 14940 \\
\hline Calypogeia peruviana Nees \& Mont. & $\mathrm{AM}, \mathrm{AT}, \mathrm{CE}$ & Wide & Tropical America & Carmo 823 \\
\hline \multicolumn{5}{|l|}{ CEPHALOZIACEAE } \\
\hline Odontoschisma denudatum (Nees) Dumort. & $\mathrm{AM}, \mathrm{AT}, \mathrm{CE}$ & Moderate & Cosmopolitan & Carmo 1042 \\
\hline Odontoschisma longiflorum (Taylor) Steph. & AT, CE & Moderate & Tropical America & Carmo 809 \\
\hline \multicolumn{5}{|l|}{ FOSSOMBRONIACEAE } \\
\hline Fossombronia porphyrorhiza (Nees) Prosk. & AT, CA, CE, PL & Wide & Tropical America & Carmo 833 \\
\hline \multicolumn{5}{|l|}{ FRULLANIACEAE } \\
\hline Frullania arecae (Spreng.) Gottsche & $\mathrm{AM}, \mathrm{AT}, \mathrm{CE}$ & Wide & Pantropical & Carmo 399 \\
\hline Frullania atrata (Sw.) Nees & $\mathrm{AM}, \mathrm{AT}$ & Wide & Tropical America & Peralta 15705 \\
\hline Frullania brasiliensis Raddi & $\mathrm{AT}, \mathrm{CE}$ & Wide & Tropical America & Carmo 1030 \\
\hline${ }^{*}$ Frullania breuteliana Gottsche & AT & Moderate & Neotropical & Peralta 15683 \\
\hline Frullania ericoides (Nees) Mont. & $\mathrm{AM}, \mathrm{AT}, \mathrm{CA}, \mathrm{CE}, \mathrm{PA}, \mathrm{PL}$ & Wide & Pantropical & Peralta 15096 \\
\hline Frullania gibbosa Nees & AM, AT, CA, CE, PL & Wide & Tropical America & Carmo 1052 \\
\hline Frullania glomerata (Lehm. \& Lindenb.) Mont. & AT, CA, CE, PL & Wide & Tropical America & Peralta 15417 \\
\hline Frullania kunzei (Lehm. \& Lindenb.) Lehm. \& Lindenb. & $\mathrm{AM}, \mathrm{AT}, \mathrm{CE}, \mathrm{PL}$ & Wide & Tropical America & Carmo 969 \\
\hline Frullania schaefer-verwimpii Yuzawa \& Hatt. & $\mathrm{AT}$ & Rare & Endemic to Brazil & Peralta 15060 \\
\hline \multicolumn{5}{|l|}{ HERBETACEAE } \\
\hline Herbertus bivittatus Spruce & $\mathrm{AM}, \mathrm{AT}$ & Moderate & Tropical America & Peralta 15639 \\
\hline${ }^{*}$ Herbertus sendtneri (Nees) A. Evans & AT & Rare & Tropical America & Peralta 15628 \\
\hline \multicolumn{5}{|l|}{ JUNGERMANNIACEAE } \\
\hline Jungermannia amoena Lindenb. \& Gottsche & $\mathrm{AM}, \mathrm{AT}$ & Moderate & Tropical America & Carmo 884 \\
\hline Jungermannia hyalina Lyell & $\mathrm{AT}, \mathrm{CE}, \mathrm{PL}$ & Moderate & Cosmopolitan & Peralta 15082 \\
\hline${ }^{*}$ Jungermannia sphaerocarpa Hook. & $\mathrm{AT}$ & Rare & Cosmopolitan & Carmo 1046 \\
\hline \multicolumn{5}{|l|}{ LEJEUNEACEAE } \\
\hline${ }^{*}$ Acanthocoleus trigonus (Nees \& Mont.) Gradst. & AT & Moderate & Neotropical & Carmo 481 \\
\hline Acrolejeunea torulosa (Lehm. \& Lindenb.) Schiffn. & $\mathrm{AM}, \mathrm{AT}, \mathrm{CE}, \mathrm{PL}$ & Wide & Tropical America & Peralta 15107 \\
\hline Anoplolejeunea conferta (C.F.W. Meissn.) A. Evans & $\mathrm{AM}, \mathrm{AT}$ & Wide & Tropical America & Carmo 804 \\
\hline${ }^{*}$ Archilejeunea auberiana (Mont.) A. Evans & AM, AT, CE & Wide & Tropical America & Peralta 15611 \\
\hline Archilejeunea parviflora (Nees) Schiffn. & $\mathrm{AM}, \mathrm{AT}, \mathrm{CE}, \mathrm{PL}$ & Wide & Tropical America & Peralta 15132 \\
\hline Brachiolejeunea leiboldiana (Gottsche \& Lindenb.) Schiffn. & $\mathrm{AT}$ & Rare & Tropical America & Carmo 400 \\
\hline${ }^{*}$ Ceratolejeunea confusa Schust. & $\mathrm{AM}, \mathrm{AT}$ & Moderate & Neotropical & Peralta 15550 \\
\hline Ceratolejeunea cornuta (Lindenb.) Schiffn. & $\mathrm{AM}, \mathrm{AT}$ & Wide & Tropical America & Carmo 1016 \\
\hline Cheilolejeunea acutangula (Nees) Grolle & $\mathrm{AM}, \mathrm{AT}, \mathrm{CE}$ & Wide & Neotropical & Peralta 15063 \\
\hline${ }^{*}$ Cheilolejeunea beyrichii (Lindenb.) Reiner & CA, CE & Rare & Endemic to Brazil & Peralta 15049 \\
\hline Cheilolejeunea clausa (Nees \& Mont.) Schust. & $\mathrm{AM}, \mathrm{AT}, \mathrm{CE}, \mathrm{PL}$ & Wide & Tropical America & Peralta 15554 \\
\hline Cheilolejeunea comans (Spruce) Schust. & $\mathrm{AM}, \mathrm{AT}$ & Rare & South Tropical America & Carmo 1028 \\
\hline Cheilolejeunea discoidea (Lehm. \& Lindenb.) Kachr. \& Schust. & $\mathrm{AT}, \mathrm{CE}, \mathrm{PL}$ & Moderate & Tropical America & Carmo 841 \\
\hline Cheilolejeunea revoluta (Herzog) Gradst. \& Grolle & $\mathrm{AT}, \mathrm{CE}$ & Rare & Neotropical & Peralta 15116 \\
\hline Cheilolejeunea rigidula (Mont.) Schust. & AM, AT, CA, CE, PL & Wide & Tropical America and Africa & Carmo 976 \\
\hline
\end{tabular}


Table 1. Cont.

\begin{tabular}{|c|c|c|c|c|}
\hline Species & Phyt. Dom. & Brazil distr. & World distr. & Voucher \\
\hline Cheilolejeunea trifaria (Reinw. et al.) Mizut. & $\mathrm{AM}, \mathrm{AT}, \mathrm{CE}, \mathrm{PL}$ & Wide & Pantropical & Carmo 898 \\
\hline Cheilolejeunea unciloba (Lindenb.) Malombe & $\mathrm{AT}, \mathrm{CE}$ & Moderate & Tropical America and Africa & Peralta 15480 \\
\hline Cheilolejeunea xanthocarpa (Lehm. \& Lindenb.) Malombe & $\mathrm{AT}, \mathrm{CE}$ & Moderate & Pantropical & Peralta 14992 \\
\hline Cololejeunea cardiocarpa (Mont.) A. Evans & $\mathrm{AM}, \mathrm{AT}, \mathrm{CE}$ & Wide & Pantropical & Peralta 14916 \\
\hline${ }^{*}$ Cololejeunea contractiloba A. Evans & $\mathrm{AM}$ & Rare & Endemic to Brazil & Carmo 1048 \\
\hline${ }^{*}$ Cololejeunea diaphana A. Evans & $\mathrm{AM}, \mathrm{AT}, \mathrm{CE}$ & Wide & Endemic to Brazil & Peralta 15180 \\
\hline Cololejeunea gracilis (Jovet-Ast.) Pócs. & AM, AT, CE & Moderate & Neotropical & Peralta 15178 \\
\hline Cololejeunea minutissima (Sm.) Schiffn. & $\mathrm{AM}, \mathrm{AT}, \mathrm{CE}, \mathrm{PL}$ & Wide & Pantropical & Peralta 15081 \\
\hline Cololejeunea subcardiocarpa Tixier & $\mathrm{AM}, \mathrm{AT}, \mathrm{CE}$ & Wide & Tropical America & Peralta 15545 \\
\hline Frullanoides liebmanniana (Lindenb. \& Gottsche) van Slageren & $\mathrm{AM}, \mathrm{AT}, \mathrm{CE}$ & Moderate & Tropical America & Carmo 1011 \\
\hline Harpalejeunea oxyphylla (Nees \& Mont.) Steph. & $\mathrm{AM}, \mathrm{AT}$ & Moderate & Tropical America & Peralta 15605 \\
\hline Harpalejeunea stricta (Lindenb. \& Gottsche) Steph. & $\mathrm{AM}, \mathrm{AT}$ & Moderate & Tropical America & Peralta 15706 \\
\hline${ }^{*}$ Lejeunea adpressa Nees & $\mathrm{AM}, \mathrm{AT}$ & Rare & Tropical America and Africa & Peralta 15455 \\
\hline *Lejeunea calcicola R.M. Schust. & AT, CE & Rare & Cosmopolitan & Peralta 15461 \\
\hline${ }^{*}$ Lejeunea cancellata Nees \& Mont. & AT, CE, PL & Wide & Argentina and Brazil & Peralta 15112 \\
\hline Lejeunea capensis Gottsche & AT & Rare & Pantropical & Peralta 15454 \\
\hline${ }^{*}$ Lejeunea caulicalyx (Steph.) E. Reiner \& Goda & $\mathrm{AM}, \mathrm{AT}, \mathrm{CE}, \mathrm{PL}$ & Wide & Tropical America & Carmo 1014 \\
\hline Lejeunea cristulata (Steph.) E. Reiner \& Goda & AT & Moderate & Endemic to Brazil & Carmo 1036 \\
\hline${ }^{*}$ Lejeunea erostrata E. Reiner \& Goda & $\mathrm{AM}$ & Rare & Endemic to Brazil & Peralta 15534 \\
\hline${ }^{*}$ Lejeunea filipes Spruce & AT, PL & Rare & Neotropical & Peralta 15140 \\
\hline Lejeunea flava (Sw.) Nees & AM, AT, CA, CE, PA, PL & Wide & Pantropical & Carmo 894 \\
\hline${ }^{*}$ Lejeunea immersa Spruce & AT & Moderate & South Tropical America & Carmo 941 \\
\hline Lejeunea laetevirens Nees \& Mont. & $\mathrm{AM}, \mathrm{AT}, \mathrm{CA}, \mathrm{CE}, \mathrm{PL}$ & Wide & Tropical America & Peralta 15206 \\
\hline Lejeunea lepida Lindenb. \& Gottsche & AT, PL & Moderate & Tropical America & Peralta 15508 \\
\hline Lejeunea oligoclada Spruce & $\mathrm{AT}$ & Moderate & Endemic to Brazil & Peralta 15090 \\
\hline Lejeunea phyllobola Nees \& Mont. & AM, AT, CA, CE, PL & Wide & Tropical America & Carmo 1024 \\
\hline * Lejeunea ruthii (A. Evans) R.M. Schust. & $\mathrm{AT}$ & Rare & Tropical America & Peralta 15484 \\
\hline${ }^{*}$ Leptolejeunea exocellata (Spruce) A. Evans & $\mathrm{AM}, \mathrm{AT}, \mathrm{CE}, \mathrm{PL}$ & Wide & Tropical America & Carmo 1001 \\
\hline Lopholejeunea nigricans (Lindenb.) Schiffn. & AM, AT, CE, PL & Wide & Pantropical & Peralta 15153 \\
\hline Lopholejeunea subfusca (Nees) Schiffn. & $\mathrm{AM}, \mathrm{AT}, \mathrm{CA}, \mathrm{CE}, \mathrm{PL}$ & Wide & Pantropical & Peralta 15505 \\
\hline Mastigolejeunea auriculata (Wilson) Schiffn. & $\mathrm{AM}, \mathrm{AT}, \mathrm{CE}, \mathrm{PL}$ & Wide & Pantropical & Peralta 15560 \\
\hline${ }^{*}$ Mastigolejeunea innovans (Spruce) Steph. & $\mathrm{AM}, \mathrm{AT}, \mathrm{CE}$ & Moderate & Neotropical & Peralta 15594 \\
\hline${ }^{*}$ Metalejeunea cucullata (Reinw. et al.) Grolle & AT & Rare & Pantropical & Peralta 14842 \\
\hline Microlejeunea bullata (Taylor) Steph. & $\mathrm{AM}, \mathrm{AT}, \mathrm{CA}, \mathrm{CE}, \mathrm{PA}, \mathrm{PL}$ & Wide & Tropical America & Peralta 14921 \\
\hline Microlejeunea epiphylla Bischl. & AM, AT, CA, CE, PL & Wide & Neotropical & Peralta 15616 \\
\hline Omphalanthus filiformis (Sw.) Nees & $\mathrm{AM}, \mathrm{AT}, \mathrm{PL}$ & Wide & Tropical America & Peralta 15537 \\
\hline Schiffneriolejeunea polycarpa (Nees) Gradst. & $\mathrm{AM}, \mathrm{AT}, \mathrm{CA}, \mathrm{CE}, \mathrm{PL}$ & Wide & Pantropical & Peralta 15064 \\
\hline Taxilejeunea isocalycina (Nees) Steph. & $\mathrm{AM}, \mathrm{AT}$ & Moderate & Tropical America & Peralta 15644 \\
\hline \multicolumn{5}{|l|}{ LEPIDOZIACEAE } \\
\hline Bazzania heterostipa (Steph.) Fulford & $\mathrm{AT}$ & Moderate & Endemic to Brazil & Peralta 15423 \\
\hline Bazzania hookeri (Lindenb.) Trevis. & AM, AT & Wide & Tropical America & Peralta 15134 \\
\hline Kurzia brasiliensis (Steph.) Grolle & $\mathrm{AM}, \mathrm{AT}, \mathrm{CE}$ & Wide & Endemic to Brazil & Peralta 15631 \\
\hline Kurzia capillaris (Sw.) Grolle & $\mathrm{AM}, \mathrm{AT}, \mathrm{CE}$ & Wide & Tropical America and Africa & Carmo 1009 \\
\hline Lepidozia cupressina (Sw.) Lindenb. & AT & Rare & Cosmopolitan & Peralta 15196 \\
\hline Lepidozia inaequalis (Lehm. \& Lindenb.) Lehm. \& Lindenb. & AT & Moderate & South Tropical America & Peralta 15405 \\
\hline Micropterygium campanense Spruce ex Reimers & AT & Rare & Tropical America & Peralta 14939 \\
\hline Paracromastigum pachyrhizum (Nees) Fulford & $\mathrm{AT}, \mathrm{CE}$ & Moderate & Tropical America & Carmo 869 \\
\hline *Pteropsiella serrulata Spruce ex Steph. & $\mathrm{AM}$ & Rare & South Tropical America & Peralta 15403 \\
\hline Telaranea nematodes (Gottshe ex Austin) M. Howe. Bull. & $\mathrm{AM}, \mathrm{AT}, \mathrm{CE}$ & Wide & Pantropical & Peralta 14909 \\
\hline \multicolumn{5}{|l|}{ LOPHOCOLEACEAE } \\
\hline *Chiloscyphus liebmannianus (Gottsche) J.J. Engel \& R.M. Schust. & $\mathrm{AM}, \mathrm{AT}, \mathrm{CE}$ & Moderate & Tropical America & Peralta 15244 \\
\hline Chiloscyphus lindmannii (Stephani) J.J. Engel \& R.M. Schust. & $\mathrm{AM}, \mathrm{AT}, \mathrm{CE}$ & Moderate & South Tropical America & Peralta 15191 \\
\hline Chyloscyphus martianus (Nees) J.J. Engel \& R.M. Schust. & $\mathrm{AM}, \mathrm{AT}, \mathrm{CE}, \mathrm{PL}$ & Wide & Tropical America and Africa & Peralta 15200 \\
\hline Chiloscyphus muricatus (Lehm.) J.J. Engel \& R.M. Schust. & $\mathrm{AT}$ & Moderate & Cosmopolitan & Peralta 15578 \\
\hline Chiloscyphus platensis (C. Massal.) J.J. Engel & $\mathrm{AT}, \mathrm{CE}$ & Moderate & South Tropical America & Peralta 14890 \\
\hline${ }^{*}$ Clasmatocolea vermicularis (Lehm.) Grolle & AT, PL & Moderate & Tropical America and Africa & Carmo 931 \\
\hline Heteroscyphus amphibolius (Nees) Schiffn. & $\mathrm{AT}, \mathrm{CE}$ & Moderate & Tropical America & Carmo 1073 \\
\hline \multicolumn{5}{|l|}{ METZGERIACEAE } \\
\hline Metzgeria dichotoma (Sw.) Nees & $\mathrm{AT}, \mathrm{CE}$ & Moderate & Tropical America & Carmo 993 \\
\hline
\end{tabular}


Table 1. Cont.

\begin{tabular}{|c|c|c|c|c|}
\hline Species & Phyt. Dom. & Brazil distr. & World distr. & Voucher \\
\hline Metzgeria furcata (L.) Dumort. & $\mathrm{AM}, \mathrm{AT}, \mathrm{CE}$ & Wide & Subcosmopolitan & Peralta 15000 \\
\hline \multicolumn{5}{|l|}{ PALLAVICINIACEAE } \\
\hline Symphyogyna aspera Steph. & $\mathrm{AM}, \mathrm{AT}, \mathrm{CE}, \mathrm{PL}$ & Wide & Tropical America & Carmo 1076 \\
\hline Symphyogyna brasiliensis (Nees) Nees \& Mont. & $\mathrm{AM}, \mathrm{AT}, \mathrm{CE}$ & Wide & Neotropical & Carmo 805 \\
\hline Symphyogyna podophylla (Thunb.) Mont. \& Nees & AT & Moderate & Tropical America and Africa & Peralta 14936 \\
\hline \multicolumn{5}{|l|}{ PLAGIOCHILACEAE } \\
\hline Plagiochila bryopterioides Spruce & AT & Rare & Tropical America & Peralta 15699 \\
\hline Plagiochila corrugata (Nees) Nees \& Mont. & $\mathrm{AM}, \mathrm{AT}, \mathrm{CE}$ & Wide & Tropical America & Carmo 1002 \\
\hline Plagiochila martiana (Nees) Lindenb. & $\mathrm{AM}, \mathrm{AT}, \mathrm{CE}, \mathrm{PL}$ & Wide & Tropical America & Peralta 15181 \\
\hline Plagiochila raddiana Lindenb. & AM, AT, CE & Wide & Tropical America & Peralta 15235 \\
\hline Plagiochila simplex (Sw.) Lindenb. & $\mathrm{AM}, \mathrm{AT}, \mathrm{CE}$ & Wide & Tropical America & Carmo 1062 \\
\hline \multicolumn{5}{|l|}{ PORELLACEAE } \\
\hline Porella brasiliensis (Raddi) Schiffn. & AT, CE & Moderate & South Tropical America & Peralta 15234 \\
\hline Porella swartziana (Weber) Trevis. & AT, CE & Moderate & Tropical America & Peralta 15547 \\
\hline \multicolumn{5}{|l|}{ RADULACEAE } \\
\hline Radula quadrata Gottsche & $\mathrm{AM}, \mathrm{AT}$ & Moderate & Cosmopolitan & Carmo 995 \\
\hline \multicolumn{5}{|l|}{ SCAPANIACEAE } \\
\hline Anastrophyllum auritum (Lehm.) Steph. & AT & Rare & Tropical America and Africa & Peralta 14984 \\
\hline Anastrophyllum piligerum (Nees) Steph. & AT & Rare & Pantropical & Peralta 15001 \\
\hline \multicolumn{5}{|l|}{ Bryophyta } \\
\hline \multicolumn{5}{|l|}{ BARTRAMIACEAE } \\
\hline Breutelia microdonta (Mitt.) Broth. & AT & Moderate & Endemic to Brazil & Peralta 15098 \\
\hline Philonotis sphaerocarpa (Hedw.) Brid. & $\mathrm{AM}, \mathrm{AT}, \mathrm{CA}, \mathrm{CE}$ & Moderate & Americas & Carmo 958 \\
\hline Philonotis uncinata (Schwägr.) Brid. & AM, AT, CA, CE, PA, PL & Wide & Cosmopolitan & Carmo 897 \\
\hline \multicolumn{5}{|l|}{ BRACHYTHECIACEAE } \\
\hline Meteoridium remotifolium (Müll. Hal.) Manuel & $\mathrm{AM}, \mathrm{AT}, \mathrm{CE}$ & Wide & Neotropical & Peralta 15704 \\
\hline Squamidium brasiliense Broth. & $\mathrm{AT}$ & Moderate & Tropical America and Africa & Carmo 870 \\
\hline * Squamidium isocladum (Renauld \& Cardot) Broth. & AT & Rare & Neotropical & Peralta 14998 \\
\hline Zelometeorium patens (Hook.) Manuel & $\mathrm{AT}, \mathrm{CE}$ & Moderate & Neotropical & Carmo 1075 \\
\hline Zelometeorium patulum (Hedw.) Manuel & $\mathrm{AM}, \mathrm{AT}, \mathrm{CE}, \mathrm{PL}$ & Wide & Neotropical & Peralta 15186 \\
\hline \multicolumn{5}{|l|}{ BRUCHIACEAE } \\
\hline Trematodon longicollis Michx. & $\mathrm{AM}, \mathrm{AT}, \mathrm{CE}, \mathrm{PA}$ & Wide & Cosmopolitan & Peralta 15169 \\
\hline \multicolumn{5}{|l|}{ BRYACEAE } \\
\hline Brachymenium acuminatum Harv. & $\mathrm{AT}, \mathrm{CE}$ & Rare & Cosmopolitan & Carmo 503 \\
\hline Brachymenium exile (Dozy \& Molk.) Bosch \& Sande Lac. & $\mathrm{AT}, \mathrm{CA}, \mathrm{CE}$ & Wide & Pantropical & Carmo 937 \\
\hline Bryum apiculatum Schwägr. & $\mathrm{AM}, \mathrm{AT}, \mathrm{CA}, \mathrm{CE}$ & Wide & Cosmopolitan & Peralta 15623 \\
\hline Bryum argenteum Broth. & $\mathrm{AM}, \mathrm{AT}, \mathrm{CA}, \mathrm{CE}, \mathrm{PA}$ & Wide & Cosmopolitan & Carmo 410 \\
\hline Bryum billarderi Schwägr. & AM, AT, CE, PA, PL & Wide & Cosmopolitan & Peralta 15068 \\
\hline Bryum caespiticium Hedw. & AT & Rare & Cosmopolitan & Peralta 15028 \\
\hline Byum coronatum Schwägr. & $\mathrm{AM}, \mathrm{AT}, \mathrm{CE}$ & Wide & Cosmopolitan & Carmo 475 \\
\hline Bryum densifolium Brid. & $\mathrm{AT}, \mathrm{CE}$ & Wide & Neotropical and Argentina & Carmo 980 \\
\hline * Bryum dichotomum Hedw. & $\mathrm{AT}, \mathrm{CE}$ & Rare & Cosmopolitan & Peralta 15078 \\
\hline Bryum huillense Welm. \& Duby & $\mathrm{AT}$ & Rare & Cosmopolitan & Carmo 974 \\
\hline Bryum limbatum Müll. Hal. & $\mathrm{AT}, \mathrm{CE}$ & Moderate & Neotropical & Peralta 15569 \\
\hline${ }^{*}$ Bryum turbinatum (Hedw.) Turner & AT & Rare & Cosmopolitan & Carmo 404 \\
\hline Rhodobryum beyrichianum (Hornsch.) Müll. Hal. & $\mathrm{AM}, \mathrm{AT}, \mathrm{CE}$ & Wide & Cosmopolitan & Peralta 15172 \\
\hline \multicolumn{5}{|l|}{ CALYMPERACEAE } \\
\hline * Calymperes afzelii Sw. & $\mathrm{AM}, \mathrm{AT}, \mathrm{CE}$ & Wide & Pantropical & Peralta 15154 \\
\hline Octoblepharum albidum Hedw. & $\mathrm{AM}, \mathrm{AT}, \mathrm{CA}, \mathrm{CE}, \mathrm{PA}, \mathrm{PL}$ & Wide & Pantropical & Peralta 14997 \\
\hline Octoblepharum cocuiense Mitt. & $\mathrm{AM}, \mathrm{AT}, \mathrm{CE}$ & Wide & Neotropical & Peralta 14906 \\
\hline Octoblepharum erectifolium Mitt. Ex R.S. Williams & $\mathrm{AM}$ & Rare & Neotropical & Peralta 15460 \\
\hline * Octoblepharum stramineum Mitt. & $\mathrm{AM}$ & Rare & Neotropical & Peralta 15017 \\
\hline Syrrhopodon gardneri (Hook.) Schwägr. & $\mathrm{AM}, \mathrm{AT}, \mathrm{CE}$ & Moderate & Pantropical & Carmo 340 \\
\hline Syrrhopodon ligulatus Mont. & AM, AT, CE & Wide & Neotropical & Peralta 14991 \\
\hline Syrrhopodon parasiticus (Brid.) Besch. & $\mathrm{AM}, \mathrm{AT}, \mathrm{CE}, \mathrm{PL}$ & Wide & Pantropical & Peralta 15157 \\
\hline Syrrhopodon prolifer Schwägr. & $\mathrm{AM}, \mathrm{AT}, \mathrm{CA}, \mathrm{CE}$ & Wide & Pantropical & Carmo 877 \\
\hline Syrrhopodon tortilis Hampe & $\mathrm{AT}$ & Moderate & Neotropical & Carmo 1067 \\
\hline \multicolumn{5}{|l|}{ CRYPHAEACEAE } \\
\hline Schoenobryum concavifolium (Griff.) Gangulee & AM, AT, CE, PA, PL & Wide & Cosmopolitan & Carmo 498 \\
\hline
\end{tabular}


Table 1. Cont.

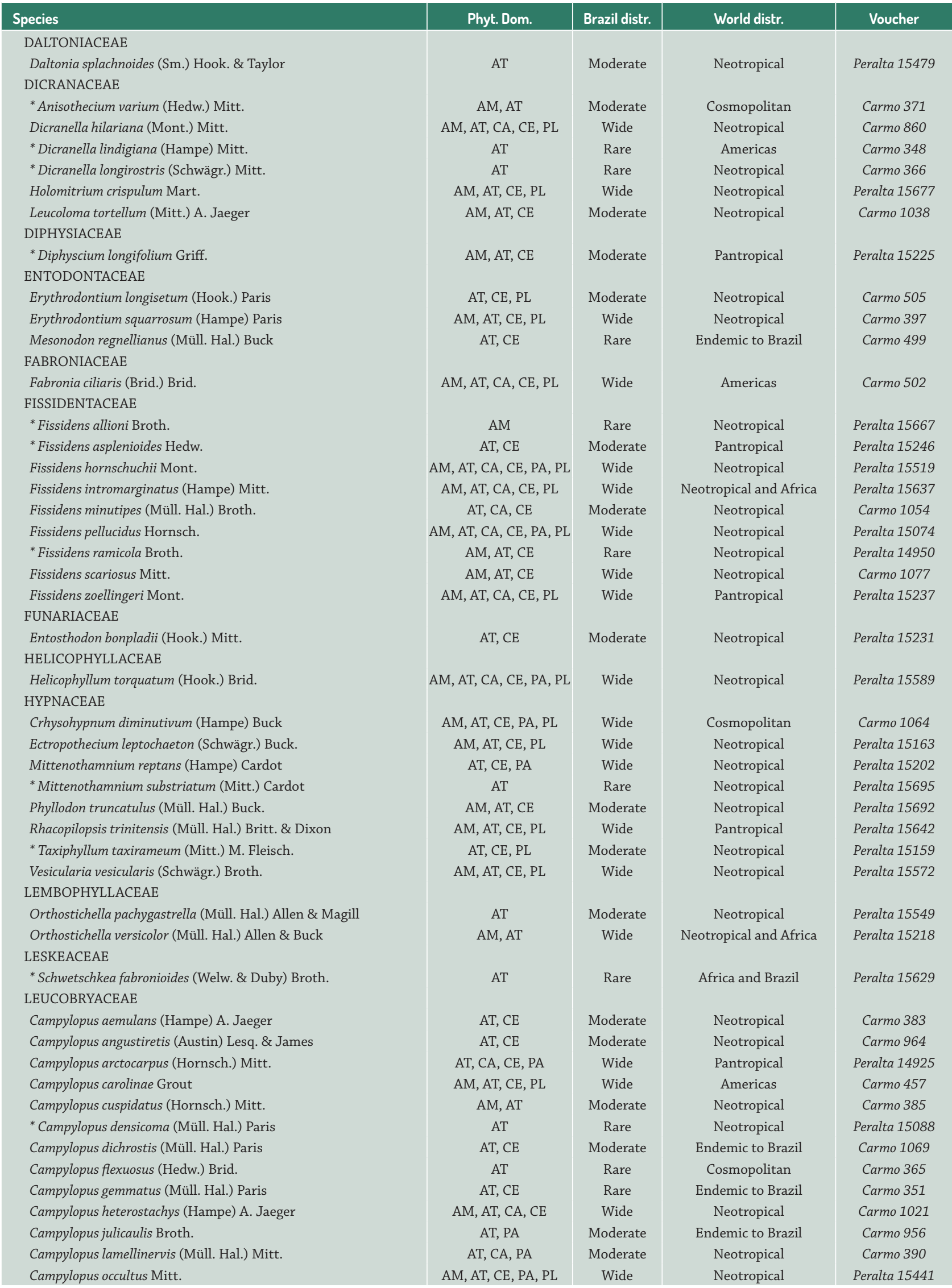


Table 1. Cont.

\begin{tabular}{|c|c|c|c|c|}
\hline Species & Phyt. Dom. & Brazil distr. & World distr. & Voucher \\
\hline Campylopus pilifer Brid. & $\mathrm{AM}, \mathrm{AT}, \mathrm{CA}, \mathrm{CE}, \mathrm{PA}$ & Wide & Neotropical & Carmo 1071 \\
\hline Campylopus richardii Brid. & AM, AT & Wide & Neotropical & Carmo 971 \\
\hline Campylopus savannarum (Müll. Hal.) Mitt. & $\mathrm{AM}, \mathrm{AT}, \mathrm{CA}, \mathrm{CE}, \mathrm{PL}$ & Wide & Pantropical & Carmo 868 \\
\hline Campylopus surinamensis Müll. Hal. & $\mathrm{AM}, \mathrm{AT}, \mathrm{CE}, \mathrm{PL}$ & Wide & Neotropical & Peralta 14945 \\
\hline Campylopus widgrenii (Müll. Hal.) Mitt. & $\mathrm{AT}$ & Rare & Endemic to Brazil & Carmo 863 \\
\hline Leucobryum clavatum Hampe & AT, CE & Moderate & Endemic to Brazil & Carmo 904 \\
\hline Ochrobryum gardneri (Müll. Hal.) Lindb. & $\mathrm{AM}, \mathrm{AT}, \mathrm{CE}, \mathrm{PL}$ & Wide & Neotropical and Africa & Peralta 15227 \\
\hline \multicolumn{5}{|l|}{ LEUCOMIACEAE } \\
\hline Leucomium strumosum (Hornsch.) Mitt. & $\mathrm{AM}, \mathrm{AT}$ & Wide & Pantropical & Peralta 15155 \\
\hline \multicolumn{5}{|l|}{ METEORIACEAE } \\
\hline * Cryptopapillaria penicillata (Dozy \& Molk.) M. Menzel & $\mathrm{AT}$ & Rare & Neotropical & Peralta 15056 \\
\hline Floribundaria flaccida (Mitt.) Broth. & $\mathrm{AM}, \mathrm{AT}, \mathrm{CE}, \mathrm{PL}$ & Wide & South America & Peralta 15215 \\
\hline Meteorium nigrescens (Hedw.) Dozy \& Molk. & $\mathrm{AM}, \mathrm{AT}, \mathrm{CE}, \mathrm{PL}$ & Wide & Neotropical & Peralta 15224 \\
\hline \multicolumn{5}{|l|}{ MNIACEAE } \\
\hline $\begin{array}{l}\text { * Epipterygium immarginatum Mitt. } \\
\text { NECKERACEAE }\end{array}$ & AT & Rare & Neotropical & Peralta 15245 \\
\hline Neckeropsis undulata (Hedw.) Reichardt & AM, AT, CA, CE, PL & Wide & Americas & Peralta 15210 \\
\hline *Pinnatella minuta (Mitt.) Broth. & $\mathrm{AM}, \mathrm{AT}$ & Rare & Pantropical & Peralta 15514 \\
\hline *Porotrichum substriatum (Hampe) Mitt. & $\mathrm{AM}, \mathrm{AT}$ & Wide & Neotropical and Africa & Peralta 15238 \\
\hline \multicolumn{5}{|l|}{ ORTHOTRICHACEAE } \\
\hline Groutiella tomentosa (Hornsch.) Wijk. \& Margad. & $\mathrm{AM}, \mathrm{AT}, \mathrm{CE}$ & Moderate & Cosmopolitan & Carmo 965 \\
\hline Macrocoma orthotrichoides (Raddi) Wijk. \& Margad. & AT & Moderate & Neotropical and India & Peralta 15475 \\
\hline Macromitrium cirrosum (Hedw.) Brid. & $\mathrm{AM}, \mathrm{AT}$ & Wide & Neotropical & Carmo 511 \\
\hline Macromitrium richardii Schwägr. & $\mathrm{AM}, \mathrm{AT}$ & Wide & Neotropical and Africa & Carmo 489 \\
\hline Schlotheimia jamesonii (Arn.) Brid. & $\mathrm{AM}, \mathrm{AT}, \mathrm{CE}, \mathrm{PA}$ & Wide & Neotropical & Carmo 509 \\
\hline \multicolumn{5}{|l|}{ PILOTRICHACEAE } \\
\hline Callicostella merkelii (Hornsch.) A. Jaeger & AM, AT, CE & Wide & Neotropical & Peralta 15570 \\
\hline Callicostella pallida (Hornsch.) Angstr. & AM, AT, CA, CE, PA, PL & Wide & Neotropical & Carmo 878 \\
\hline${ }^{*}$ Cyclodictyon varians (Sull.) Kuntze & $\mathrm{AM}, \mathrm{AT}, \mathrm{CE}$ & Moderate & Americas & Peralta 15646 \\
\hline Trachyxiphium saxicola (R.S. Willia) Vaz-Imbassahy \& Costa & AT, CE & Moderate & Neotropical & Peralta 15597 \\
\hline \multicolumn{5}{|l|}{ POLYTRICHACEAE } \\
\hline Itatiella denudata (Merril) Bell \& Hyvönen & CE & Rare & Endemic to Brazil & Carmo 1044 \\
\hline Pogonatum pensilvanicum (Bartr. ex Hedw.) P. Beauv. & AT, CE & Moderate & Americas & Carmo 358 \\
\hline Polytrichum angustifolium Mitt. & AT, PA & Moderate & Endemic to Brazil & Carmo 822 \\
\hline Polytrichum commune L. ex Hedw. & $\mathrm{AM}, \mathrm{AT}, \mathrm{CE}$ & Wide & Cosmopolitan & Carmo 386 \\
\hline Polyrichum juniperinum Wild. ex Hedw. & $\mathrm{AM}, \mathrm{AT}, \mathrm{CE}$ & Wide & Cosmopolitan & Carmo 966 \\
\hline \multicolumn{5}{|l|}{ POTTIACEAE } \\
\hline Hyophila involuta (Hook.) A. Jaeger & AM, AT, CA, CE, PA, PL & Wide & Cosmopolitan & Carmo 816 \\
\hline${ }^{*}$ Tortella linearis (Sw. ex F. Weber \& D. Mohr) R.H. Zander & AT & Rare & Neotropical & Peralta 15617 \\
\hline \multicolumn{5}{|l|}{ PTERIGYNANDRACEAE } \\
\hline \multicolumn{5}{|l|}{ PTEROBRYACEAE } \\
\hline Henicodium geniculatum (Mitt.) Buck & $\mathrm{AM}, \mathrm{AT}, \mathrm{CE}, \mathrm{PL}$ & Wide & Pantropical & Peralta 15150 \\
\hline Jaegerina scariosa (Lorentz) Arzeni & AM, AT, CE, PL & Wide & Pantropical & Carmo 915 \\
\hline Orthostichidium quadrangulare (Schwägr.) Allen & & Moderate & Tropical America and Africa & Peralta 15661 \\
\hline \multicolumn{5}{|l|}{ PYLAISIADELPHACEAE } \\
\hline Isopterygium affusum Mitt. & AM, AT, CE & Moderate & Neotropical & Carmo 919 \\
\hline Isopterygium tenerifolium Mitt. & AM, AT, CE & Wide & Neotropical & Carmo 874 \\
\hline Isopterygium tenerum (Sw.) Mitt. & AM, AT, CA, CE, PA, PL & Wide & Cosmopolitan & Peralta 14888 \\
\hline Wijkia flagellifera (Broth.) H.A. Crum & AT & Moderate & Neotropical & Peralta 15139 \\
\hline \multicolumn{5}{|l|}{ RACOPILACEAE } \\
\hline Racopilum tomentosum (Hedw.) Brid. & AM, AT, CE, PA, PL & Wide & Cosmopolitan & Carmo 417 \\
\hline
\end{tabular}


Table 1. Cont.

\begin{tabular}{|c|c|c|c|c|}
\hline Species & Phyt. Dom. & Brazil distr. & World distr. & Voucher \\
\hline * Tisserantiella minutissima (Mitt.) R.H. Zander & CE & Rare & Bolivia and Brazil & Peralta 14969 \\
\hline $\begin{array}{l}\text { RHACOCARPACEAE } \\
\text { Rhacocarpus purpurascens (Brid.) Müll. Hal. } \\
\text { RHYZOGONIACEAE }\end{array}$ & $\mathrm{AT}$ & Moderate & Cosmopolitan & Carmo 1066 \\
\hline $\begin{array}{l}\text { Pyrrhobryum spiniforme (Hedw.) Mitt. } \\
\text { SEMATOPHYLLACEAE }\end{array}$ & $\mathrm{AM}, \mathrm{AT}, \mathrm{CE}, \mathrm{PA}$ & Wide & Cosmopolitan & Peralta 15138 \\
\hline Aptychopsis pungifolia (Hampe) Borth. & AT & Moderate & Endemic to Brazil & Carmo 902 \\
\hline Aptychopsis subpungifolia (Broth.) Broth. & $\mathrm{AT}, \mathrm{CE}$ & Moderate & Endemic to Brazil & Carmo 1050 \\
\hline Colobodontium vulpinum (Mont.) S.P. Churchill \& Buck & AM, AT, CE & Wide & Neotropical & Carmo 873 \\
\hline Donnellia commutata (Müll. Hal.) Buck & $\mathrm{AM}, \mathrm{AT}, \mathrm{CE}, \mathrm{PL}$ & Moderate & Neotropical & Peralta 14956 \\
\hline Potamium lonchophyllum (Mont.) Mitt. & $\mathrm{AM}, \mathrm{AT}, \mathrm{CE}$ & Moderate & Neotropical & Peralta 14953 \\
\hline Sematophyllum beyrichii (Hornsch.) Broth. & AT & Moderate & Neotropical & Carmo 810 \\
\hline Sematophyllum galipense (Müll. Hal.) Mitt. & $\mathrm{AM}, \mathrm{AT}, \mathrm{CE}$ & Wide & Neotropical & Carmo 946 \\
\hline * Sematophyllum oedophysidium Buck & $\mathrm{AM}, \mathrm{AT}$ & Rare & Endemic to Brazil & Carmo 912 \\
\hline Sematophyllum subpinnatum (Brid.) Britt. & AM, AT, CA, CE, PA, PL & Wide & Pantropical & Carmo 1018 \\
\hline $\begin{array}{l}\text { Sematophyllum subsimplex (Hedw.) Mitt. } \\
\text { SPHAGNACEAE }\end{array}$ & AM, AT, CA, CE, PL & Wide & Neotropical & Carmo 1060 \\
\hline Sphagnum aciphyllum Müll. Hal. & AT, PA & Moderate & South Tropical America & Carmo 950 \\
\hline Sphagnum brasiliense Warnst. & $\mathrm{AT}$ & Rare & Endemic to Brazil & Carmo 959 \\
\hline Sphagnum capillifolium (Ehrh.) Hedw. & $\mathrm{AM}, \mathrm{AT}, \mathrm{PA}$ & Moderate & Cosmopolitan & Peralta 15062 \\
\hline${ }^{*}$ Sphagnum contortulum H.A. Crum & $\mathrm{AT}$ & Rare & Endemic to Brazil & Peralta s.n. (SP439583) \\
\hline Sphagnum cuspidatum Ehrh. ex Hoffm. & AT, PA & Moderate & Cosmopolitan & Peralta 14949 \\
\hline Sphagnum cyclophyllum Sull. \& Lesq. & AT & Moderate & Neotropical & Carmo 906 \\
\hline * Sphagnum delamboyense Schäf-Verw. & $\mathrm{CE}$ & Rare & Endemic to Brazil & Carmo 923 \\
\hline * Sphagnum dimorphophyllum Crum \& Buck & $\mathrm{AM}$ & Rare & Endemic to Brazil & Carmo 921 \\
\hline Sphagnum divisum H.A. Crum & AT, CE & Moderate & Endemic to Brazil & Peralta 15229 \\
\hline Sphagnum geraisense H.A. Crum & $\mathrm{AT}$ & Rare & Endemic to Brazil & Carmo 821 \\
\hline Sphagnum longistolo Müll Hal. & $\mathrm{AT}$ & Moderate & Peru and Brazil & Peralta 15055 \\
\hline Sphagnum magellanicum Brid. & $\mathrm{AM}, \mathrm{AT}, \mathrm{CE}$ & Wide & Cosmopolitan & Peralta 15464 \\
\hline * Sphagnum ovalifolium Warnst. & CE & Rare & Endemic to Brazil & Carmo 968 \\
\hline Sphagnum perichaetiale Hampe & $\mathrm{AM}, \mathrm{AT}, \mathrm{CE}, \mathrm{PA}$ & Wide & Cosmopolitan & Peralta 15428 \\
\hline Sphagnum platyphylloides Warnst. & $\mathrm{AT}, \mathrm{CE}$ & Rare & Endemic to Brazil & Peralta 15069 \\
\hline * Sphagnum pseudoramulinum H.A. Crum & $\mathrm{AT}$ & Rare & Endemic to Brazil & Carmo 859 \\
\hline Sphagnum ramulinum Warnst. & AT, CE & Rare & Endemic to Brazil & Carmo 881 \\
\hline${ }^{*}$ Sphagnum sparsum Hampe & $\mathrm{AT}$ & Rare & Neotropical & Carmo 405 \\
\hline Sphagnum submedium Warnst. & AT & Rare & Endemic to Brazil & Carmo 977 \\
\hline Sphagnum subsecundum Nees & $\mathrm{AM}, \mathrm{AT}$ & Wide & Cosmopolitan & Carmo 865 \\
\hline Sphagnum tabuleirense O. Yano \& H.A. Crum & AT, CE, PA & Rare & Endemic to Brazil & Peralta 14962 \\
\hline Sphagnum tenerum Sull. \& Lesq. & $\mathrm{AT}$ & Moderate & Cosmopolitan & Peralta 15492 \\
\hline Sphagnum turgens Warnst. & $\mathrm{AT}$ & Rare & Endemic to Brazil & Carmo 388 \\
\hline * Sphagnum vitalii H.A. Crum & $\mathrm{AT}$ & Rare & Endemic to Brazil & Peralta 14857 \\
\hline STEREOPHYLLACEAE & & & & \\
\hline Entodontopsis leucostega (Brid.) Buck \& Ireland & $\mathrm{AM}, \mathrm{AT}, \mathrm{CA}, \mathrm{CE}, \mathrm{PL}$ & Wide & Cosmopolitan & Carmo 1047 \\
\hline Pilosium clorophyllum (Hornsch.) Müll. Hal. & AM, AT, CE, PL & Wide & Neotropical & Peralta 15497 \\
\hline Stereophyllum radiculosum (Hook.) Mitt. & AM, AT, CE, PL & Wide & Pantropical & Carmo 991 \\
\hline $\begin{array}{l}\text { SYMPHYODONTACEAE } \\
\text { Symphyodon imbricatifolius (Mitt.) S.P. Churchill } \\
\text { THUIDIACEAE }\end{array}$ & AT, CE & Rare & Neotropical & Peralta 15566 \\
\hline Pelekium muricatulum (Hampe) A. Touw & $\mathrm{AT}, \mathrm{CE}$ & Moderate & South Tropical America & Peralta 15018 \\
\hline Pelekium schistocalyx (Müll. Hal.) A. Touw & AM, AT, CE, PL & Wide & Neotropical and Florida & Carmo 916 \\
\hline Thuidium delicatulum (Hedw.) Schimp. & $\mathrm{AM}, \mathrm{CE}, \mathrm{AT}$ & Wide & Cosmopolitan & Peralta 15148 \\
\hline Thuidium pseudoprotensum (Müll. Hal.) Mitt. & $\mathrm{AT}$ & Moderate & Neotropical & Peralta 15009 \\
\hline Thuidium tomentosum Schimp. & AM, AT, CE, PL & Wide & Neotropical & Peralta 15700 \\
\hline
\end{tabular}


(nine species). Fifty-six species (19\%) were considered new ocurrences in the state, while $14 \%$ of bryophytes species (41 species) were considered endemic to Brazil.

Other studies in high altitude areas of Minas Gerais State, also found greater species richness of mosses than liverworts (Yano \& Carvalho 1995; Yano \& Peralta 2009; Yano \& Peralta 2011a; b; Luizi-Ponzo et al. 2013). This is probably due to mosses having more morphologically complex gametophytes and sporophytes, which increases the possibilities for occupation of varied environments. Furthermore, mosses are more resistant to dessication than most of liverworts (Goffinet et al. 2009), thereby they can occur and predominate in areas where the availability of water is more restricted, and conditons of moisture and rainfall are relatively low, unlike the Atlantic Forest where the species richness of mosses is lower than that of liverworts (Gradstein et al. 2001).

Sphagnaceae was the most species rich moss family and, in including a number of Brazilian endemics and new occurrences, of significant importance to Serra da Canastra (Tab. 1). Although this family is often associated with watercourses and water-soaked soils (Schofield 1985), it can also be found on rocky and extremely dry surfaces, with soil and rocks as their main substrates.

Leucobryaceae (21 species) and Bryaceae (13 species) were the bryophyte families with the greatest richness in high altitudes areas in Minas Gerais (Yano \& Carvalho 1995; Yano \& Peralta 2011b). This is supported by the fact that representatives of these families are constantly found in mountain regions (Gradstein et al. 2001). These families have in common an acrocarpous growth habit of the gametophyte, and are found in more exposed areas, such as the vast rocky fields of the Cerrado (Bastos \& Vilas Bôas-Bastos 2008). Sematophyllaceae and Hypnaceae were the most represented pleurocarpous moss families and are widely distributed worldwide, mainly in the tropics, and are very diverse morphologically (Buck 1998; Ramsay et al. 2002; Ireland \& Buck 2009).

The family of liverworts with the greatest species richness was Lejeuneaceae, which has 56 genera and 285 species in Brazil (Costa et al. 2015). In the present study the family accounted for $47 \%$ of the liverworts found, and has frequently been among the most abundant families of liverworts in other studies on mountainous areas of Minas Gerais (Yano \& Carvalho 1995; Yano \& Peralta 2009; Yano \& Peralta 2011a; b; Luizi-Ponzo et al. 2013). This family is known to have originated relatively recently during bryophyte evolution, and includes many independent diversifications resulting in great morphological variation, several forms of substrate occupation and different taxonomic conceptulizations which have resulted in this great diversity presented by the family (Groth-Malonek et al. 2004).

Phaeoceros laevis was the only species of hornwort (Anthocerotophyta) found in Serra da Canastra National
Park, where it was collected growing on rocks among rocky outcrops near streams of the lower portion of the Casca D'anta waterfall. Only one of the previous studies in Minas Gerais also found this species (Yano \& Peralta 2011b). In Serra do Cipó, this species was found growing on soil and was associated with the thallose liverwort Fossombronia porphyrorhyza.

\section{Substrate colonization}

Regarding substrate colonization, a predominance of rupicolous bryophytes was observed in Serra da Canastra National Park. Among the 1,177 bryophyte samples collected, 406 were on rocky substrates, 356 were corticolous, 349 terrestrial, 27 epiphyllous and 18 epixylic (Fig. 1). Mosses that were found growing submerged or under the water were considered aquatic and, with other different substrates types (termite mound, litter), represented 21 samples (Fig. 1).

In other studies conducted in Minas Gerais, species of bryophytes showed a preference for corticolous substrates (Yano \& Peralta 2011b; Luizi-Ponzo et al. 2013), such as growing on the trunks of living trees. In their study focusing on gallery forests of Serra do Cipó, Souza \& Câmara (2015) also observed a preference for corticicolous substrates and explanained this finding on the abundance of this substrate in the area, but concluded that ecological studies are needed to verify this hypothesis. Following this reasoning, the large amount of rupicolous species in Serra da Canastra National Park is likely due to the presence of many rocks, especially in areas near waterfalls and in rocky fields, since the methodology of collection was the same for all vegetation types. However, as with the gallery forest of Serra do Cipó, ecological study is necessary to conclude this hypothesis.

Our study collected 27 samples (Fig. 1) of epiphyllous bryophytes, which are those species that grow on living leaves. The family Lejeuneaceae was the only family found

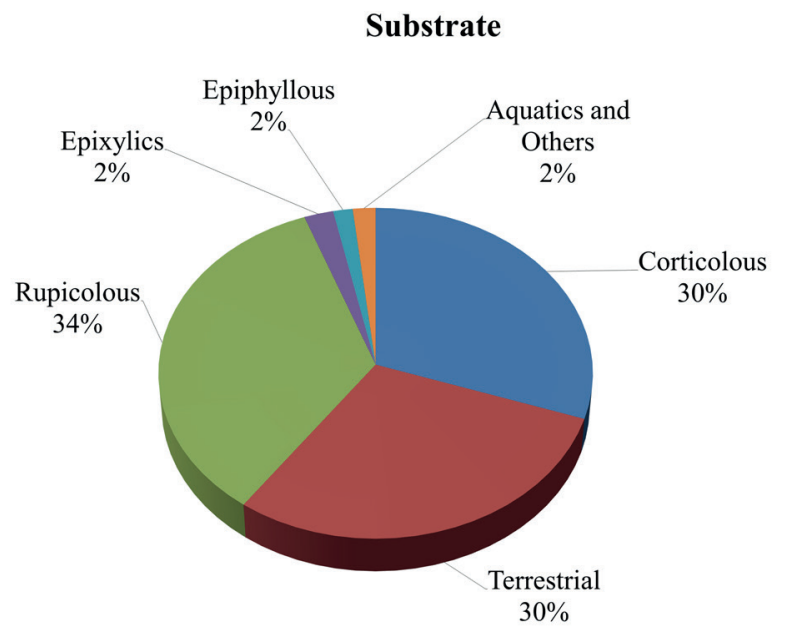

Figure 1. Percentage of samples obtained from each substrate. 


\section{Worldwide distribution}

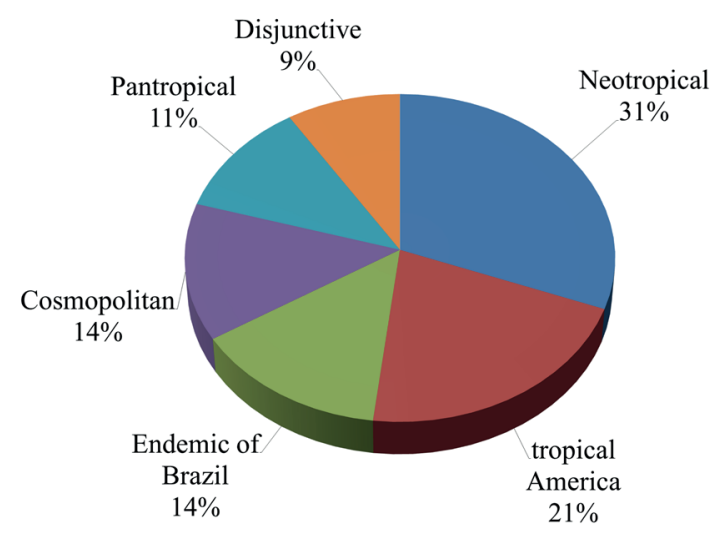

Figure 2. Percentage of bryophytes species obtained and their worldwide distribution.

growing on this type of substrate in Serra da Canastra National Park. According to Gradstein et al. (2001), this family can colonize many types of substrates, but the epiphyllous habit is common in preserved native areas, which highlights the exigency for the protection of Serra da Canastra National Park.

\section{Worldwide and Brazilian distribution}

Concerning worldwide distribution, a predominance of Neotropical species was observed in Serra da Canastra National Park, with 89 species. This was followed by 61 tropical America species, 41 Brazilian endemic species, 39 cosmopolitan species, 33 pantropical species and 26 with disjunct distributions with the Andes, Africa and other countries of South America (Fig. 2) (Tab. 1).

Most of these species (132) have a wide geographic distribution in Brazil, accounting for $46 \%$ of bryophytes found in the area (Tab. 1). These species are typically generalists and easily occupy a variety of environments and several types of substrates. Examples of such mosses (Bryophyta) include Bryum argenteum, Hyophila involuta, Octoblepharum albidum, Sematophyllum subpinnatum and Syrrhopodon prolifer. Example of such liverworts (Marchantiophyta) include Frullania ericoides and Lejeunea flava (Forzza et al. 2010). Ninety species (31\%) had a moderate distribution and 67 (23\%) were considered rare (Tab. 1).

We found 56 new species for the state of Minas Gerais, which represents $19 \%$ of the total bryophyte species collected. Among these, 36 species (64\%) have a "rare" distribution in Brazil, 13 (23\%) a "moderate" distribution and seven (13\%) a "wide" distribution (Tab. 1). Campylopus densicoma was first recorded for Minas Gerais and has a "rare" distribution, being previously found only in Rio de Janeiro, and is considered an endangered species (Costa et al. 2013).
The data obtained through this study show that the Serra da Canastra National Park is an important area for bryophyte diversity and conservation, especially since there remain many species to be discovered.

\section{Phytogeographic domains}

Serra da Canastra National Park is located in the Cerrado domain (IBDF 1981). However, most of the species (62) found in the park are considered exclusive to the Atlantic Forest domain, such as Bryum caespiticium, Squamidium brasiliense, Syrrhopodon tortilis and Wijkia flagellifera for Bryophyta (Souza \& Câmara 2015) and Bazzania heterostipa, Calypogeia grandistipula, Frullania schaefer-verwimpii and Lejeunea cristulata for Marchantiophyta (Yano 2008).

Moreover, the present study also found some Brazilian endemic species, which had so far been only recorded for the Amazon Rainforest domain, such as Cololejeunea contractiloba and Lejeunea erostrata of Marchantiophyta, and Sphagnum dimorphophyllum for Bryophyta (Costa et al. 2011). Perhaps these results are due to gaps in collections of material from areas of the Brazilian Highlands, such as Serra da Canastra (Gradstein et al. 2001; Costa et al. 2011), which is reflected in the great number of new occurrences observed in this study.

Some typical Cerrado species were found, such as Riccardia regnelli, Mesonodon regnellianus, Campylopus dichrostis, Leucobryum clavatum and Jonesiobryum cerradense, as well as some species exclusive to the Cerrado, for example, Itatiella denudata, Sphagnum delamboyense and Sphagnum garysmithii (Costa et al. 2011).

The two species endemic to Serra da Canastra National Park, Itatiella denudata and Sphagnum geraisense, were recollected during this study reinforcing the importance of maintaining Serra da Canastra National Park for the conservation of endemic species (Romero \& Nakajima 1999) as well as the great diversity of other bryophytes found in this study.

This study has provided new information on bryophyte richness, occurrence, occupied phytophysiognomies and worldwide and Brazilian distributions, particularly those occurring in mountain areas of the Brazilian Highlands, where more floristic studies on bryophytes are needed. The large number of species found in the park (289 spp.), accounting for approximately $38 \%$ of the species known in Minas Gerais and 19\% in Brazil, the new records for this state (56 spp.) and the number of Brazilian endemic species (41 spp.), stress the importance of performing floristic inventories in Brazil and maintaining Serra da Canastra National Park for conservation as a biological reserve.

\section{Acknowledgments}

We acknowledge the Botanical Institute of São Paulo (Instituto Botânico de São Paulo) and the Bryology Section 
(Seção de Briologia) for the support of all the necessary equipment throughout the study. We also acknowledge CAPES for financial support and ICMBIO for the license $\mathrm{n}^{\circ}$ 39601-2 granted collecting in the area.

\section{References}

Ballejos J, Bastos CJP. 2009. Musgos Pleurocárpicos do Parque Estadual das Sete Passagens, Miguel Calmon, Bahia, Brasil. Hoehnea 36: 479-495.

Bastos CJP, Vilas Bôas-Bastos SB. 2008. Musgos Acrocárpicos e Cladocárpicos (Bryophyta) da Reserva Ecológica da Michelin, Igrapiúna, Bahia, Brasil. Stientibus Série Ciências Biológicas 8: 275-279.

Bastos CJP, Yano O, Vilas Bôas-Bastos SB. 2000. Briófitas de Campos rupestres da Chapada Diamantina, Estado da Bahia, Brasil. Revista Brasileira de Botânica 23: 357-368.

Bates JW. 2009. Mineral nutrition and substratum ecology. In: Goffinet B, Shaw AJ. (eds.) Bryophyte Biology. 2nd. edn. New York, Cambdrige University Press. p. 299-356.

Bordin J, Yano O. 2013. Fissidentaceae (Bryophyta) do Brasil. Boletim do Instituto de Botânica 22: 1-72.

Buck WR. 1998. Pleurocarpous Mosses of the West Indies. Memoirs of The New York Botanical Garden 1: 1-401.

Câmara PEAS. 2008a. Musgos pleurocárpicos das matas de galeria da Reserva Ecológica do IBGE, RECOR, Distrito Federal, Brasil. Acta Botanica Brasilica 22: 573-581.

Câmara PEAS. 2008b. Musgos acrocárpicos das Matas de Galeria da Reserva Ecológica do IBGE, RECOR, Distrito Federal, Brasil. Acta Botanica Brasilica 22: 1027-1035.

Câmara PEAS, Costa DP. 2006. Hepáticas e antóceros das matas de galeria da Reserva Ecológica do IBGE, RECOR, Distrito Federal, Brasil. Hoehnea 33: $79-87$.

Carvalho-Silva M, Guimarães EF. 2009. Piperaceae do Parque Nacional da Serra da Canastra, Minas Gerais, Brasil. Boletim de Botânica da Universidade de São Paulo 27: 235-245.

Castro NMCF, Pôrto KC, Yano O, Castro AAJF. 2002. Levantamento florístico de bryopsida de cerrado e mata ripícola do parque nacional de Sete Cidades, Piauí, Brasil. Acta Botanica Brasilica 16: 61-76.

Costa DP, Peralta DF. 2015. Briófitas In: Lista de Espécies da Flora do Brasil. Jardim Botânico do Rio de Janeiro. http://floradobrasil.jbrj. gov.br/jabot/floradobrasil/FB128472. 19 Aug. 2015.

Costa DP, Pôrto KC, Luizi-Ponzo AP, et al. 2011. Synopsis of the Brazilian moss flora: checklist, distribution and conservation. Nova Hedwigia 93: 277-334

Costa DP, Fernandez EP, Monteiro NP, Messina T. 2013. Dicranaceae. In: Martinelli G, Moraes MA. (eds.) Livro Vermelho da Flora do Brasil. Rio de Janeiro, CNCFLORA. p. 477-478.

Costa DP, Bastos CJP, Schäfer-Verwimp A. 2015. Lejeuneaceae In: Lista de Espécies da Flora do Brasil. Jardim Botânico do Rio de Janeiro. http://www.floradobrasil.jbrj.gov.br/jabot/floradobrasil/FB97355. 19 Aug. 2015.

Couto Jr AFC, Souza VV, Junior OAC, et al. 2010. Integração de parâmetros morfométricos e imagem aster para a delimitação das fitofisionomias da Serra da Canastra, Parque Nacional da Serra da Canastra, MG. Revista Brasileira de Geomorfologia 11: 57- 68.

Crandall-Stotler B, Stotler RE, Long DG. 2009. Morphology and classification of the Marchantiophyta. In: Goffinet B, Shaw AJ. (eds.) Bryophyte Biology. 2nd. edn. New York, Cambdrige University Press. p. 1-54.

Forzza RC, Leitman PM, Costa AF, et al. 2010. Introdução. In: Forzza RC, Baumgratz JFA, Bicudo CEM, et al. (eds.) Lista de espécies da Flora do Brasil. Jardim Botânico do Rio de Janeiro. Vol. 1. Rio de Janeiro, Jardim Botânico do Rio de Janeiro.

Frahm JP. 1991. Dicranaceae: Campylopodioideae, Paraleucobryoideae. Flora Neotropica Monograph 54: 1-237.

Giulietti AM, Menezes NL, Pirani JR, Meguro M, Wanderley MGL. 1987. Flora da Serra do Cipó, Minas Gerais: caracterização e lista das espécies. Boletim de Botânica da Universidade de São Paulo 9: 1-151.
Goffinet B, Buck WR, Shaw AJ. 2009. Morphology, anatomy and classification of the Bryophyta. In: Goffinet B, Shaw AJ. (eds.) Bryophyte Biology. 2nd. edn. New York, Cambdrige University Press. p. 56-138.

Gradstein SR, Costa DP. 2003. The Hepaticae and Anthocerotae of Brazil. Memoirs of The New York Botanical Garden 87: 1-318.

Gradstein SR, Churchill, SP, Salazar-Allen N. 2001. Guide to the Bryophytes of Tropical America. Memoirs of The New York Botanical Garden 86: 1-577.

Groth-Malonek M, Heinrichs J, Schneider H, Gradstein SR. 2004. Philogenetic relationships in the Lejeuneaceae (Hepaticae) inferred using ITS sequences of nuclear ribosomal DNA. Organisms, Diversity and Evolution 4: 51-57.

Harley RM, Simmons NA. 1986. Florula of Mucugê. Chapada Diamantina - Bahia, Brazil. Kew, Royal Botanical Garden.

Holmgren PK, Keuken W, Schofield EK. 1981. Index Herbariorum. Part I. The herbaria of the world. 7th. edn. Utrecht/Antwerpen, Bohn, Scheltema \& Holkema.

IBDF - Instituto Brasileiro de Desenvolvimento Florestal. 1981. Plano de manejo. Parque Nacional da Serra da Canastra. Brasília, IBDF.

Ireland RR, Buck WR. 2009. Some Latin American Genera of Hypnaceae. Smithsonian Contributions to Botany 93: p. 1-97.

Luizi-Ponzo AP, Siviero TS, Amorim ET, et al. 2013. Briófitas do Parque Estadual do Ibitipoca no Herbário Prof. Leopoldo Kriegeer. In: Forzza RC, Neto LM, Salimena FRG, Zappi D. (eds.) Flora do Parque Estadual do Ibitipoca e seu entorno. Vol. 4. 1st. edn. Juiz de Fora, Editora UFJF. p. 95-122.

Pirani JR, Giulietti AM, Mello-Silva R, Meguro M. 1994. Checklist and patterns of geographic distribution of the vegetation of Serra do Ambrósio, Minas Gerais, Brazil. Revista Brasileira de Botânica 17: 133-147.

Ramsay HP, Schofield WB, Tan BC. 2002. The family Sematophyllaceae (Bryopsida) in Australia. Part 1: Introduction, family data, key to genera and the genera Wijkia, Acanthorrynchium, Trismegistia and Sematophyllum. The Journal of Hattori Botanical Laboratory 92: 1-50.

Renzaglia KS, Villarreal JC, Duff RJ. 2009. New insights into morphology, anatomy and systematics of hornworts. In: Goffinet B, Shaw AJ. (eds.) Bryophyte Biology. 2nd. edn. New York, Cambdrige University Press. p. 139-171.

Robbins RG. 1952. Bryophyte Ecology of a Dune Area in New Zealand. Vegetatio, Acta Geobotanica 4: 1-31.

Romero R, Nakajima JN. 1999. Espécies endêmicas do Parque Nacional da Serra da Canastra, Minas Gerais. Revista Brasileira de Botânica 22: $259-265$

Schofield WB. 1985. Introduction to Bryology. New York, Macmillan Publisching Company.

Sharp AJ, Crum H, Eckel P. 1994. The Moss Flora of Mexico. Memoirs of The New York Botanical Garden 69: 1-1113.

Souza A, Costa M. 2011. Parque Nacional da Serra da Canastra. http:// www.serradacanastra.com.br/. 19 Aug. 2015.

Souza RV, Câmara PEAS. 2015. Survey of the bryophytes of a gallery forest in the National Park of Serra do Cipó, Minas Gerais, Brazil. Acta Botanica Brasilica 29: 24-29.

Stannard BL. 1995. Flora of the Pico das Almas, Chapada Diamantina Bahia, Brazil. London, Kew, Royal Botanic Gardens.

Valente EB, Pôrto KC. 2006. Hepáticas (Marchantiophyta) de um fragmento de Mata Atlântica na Serra da Jibóia, município de Santa Terezinha, BA, Brasil. Acta Botanica Brasilica 20: 433-441.

Valente EB, Pôrto KC, Bastos CJP. 2011. Checklist of bryophytes of Chapada Diamantina, Bahia, Brazil. Boletim do Instituto de Botânica 21: 111-124.

Valente EB, Pôrto KC, Bastos CJP. 2013. Species Richness and Distribution of bryophythes within different phytophysiognomies in the Chapada Diamantina region of Brazil. Acta Botanica Brasilica 27: 294-310.

Vilas Bôas-Bastos SB, Bastos CJP. 1998. Briófitas de uma área de cerrado no município de Alagoinhas, Bahia, Brasil. Tropical Bryology 15: 101-110.

Yano O. 2008. Catálogo de antóceros e hepáticas brasileiros: literatura original, basiônimo, localidade-tipo e distribuição geográfica. Boletim do Instituto de Botânica 19: 1-110. 
Yano O, Carvalho AB. 1995. Briófitas da Serra da Piedade, Minas Gerais, Brasil. In: Anais do 9o Congresso da Sociedade Botânica de São Paulo. São Paulo, Universidade Estadual Paulista. p.15-25.

Yano O, Peralta DF. 2009. Flora de Grão-Mogol, Minas Gerais. Briófitas (Bryophyta e Marchantiophyta). Boletim de Botânica da Universidade de São Paulo 27: 1-26.
Yano O, Peralta DF. 2011a. Bryophytes from Serra de São José, Tiradentes, Minas Gerais, Brasil. Boletim de Botânica da Universidade de São Paulo 21: 141-172.

Yano O, Peralta DF. 2011b. Flora da Serra do Cipó, Minas Gerais: Briófitas (Anthocerotophyta, Bryophyta e Marchantiophyta). Boletim de Botânica da Universidade de São Paulo 29: 135-211. 